\title{
Mycobacterium Tuberculosis Infection Post Total Knee Arthroplasty in a Systemic Lupus Erythematosus (SLE) patient
}

\author{
Arieff $\mathrm{AA}^{1 *}$, Fabian Chong ${ }^{2}$, Fauzlie $\mathrm{Y}^{2}$, Faizah $\mathrm{A}^{3}$ and Sivapathasundaram $\mathrm{N}^{2}$ \\ ${ }^{1}$ Department of Orthopaedic, Hospital Tawau, Malaysia \\ ${ }^{2}$ Department of Orthopaedic, Hospital Melaka, Malaysia \\ ${ }^{3}$ Department of Pathology, Hospital Melaka, Malaysia \\ *Corresponding author: Arieff AA, Department of Orthopaedic, Hospital Tawau, Malaysia
}

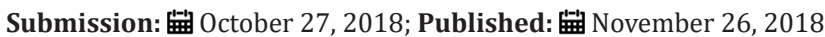

\begin{abstract}
Background: A systemic lupus erythematosus (SLE) patient presented with right knee swelling after right total knee arthroplasty, which eventually turned out to be Mycobacterium tuberculosis infection. She was successfully treated with debridement and retention of implant, together with commencement of anti-tuberculosis medications.

Case report: A 58 years-old SLE patient presented with acute, painful right knee swelling 4 months after undergoing right total knee arthroplasty. Upon examination, her right knee was diffusely swollen and warm. The initial blood parameters were normal. She was empirically started on intravenous ceftriaxone. As there was no sign of regression, she underwent arthrotomy washout and exchange of polyethylene insert, during which intraoperatively it was noted that there was presence of reactive synovitis. Post-operatively, she developed a pus-draining sinus and thus underwent another debridement with implant retention. The subsequent culture and histopathological examination of the synovial tissue confirmed the diagnosis of Mycobacterium tuberculosis infection. The patient was started on anti-tuberculosis therapy and showed marked improvement in terms of clinical parameters and functional outcomes.
\end{abstract}

Conclusion: This is a rare case of Mycobacterium infection post total knee arthroplasty. It was diagnosed by confirmation of both culture and histopathological examination of synovial tissue. The relationship between SLE and TB infection and the possibility of reactivation of pre-existing TB was explored. Surgical debridement with adjuvant anti-tuberculosis therapy remains as the mainstay treatment of such prosthetic joint infection.

Keywords: Mycobacterium tuberculosis; Prosthetic joint; Total knee arthroplasty; Systemic lupus erythematosus

\section{Introduction}

A prosthetic joint infection remains as one of the most dreaded complication's faced by Orthopaedic surgeons following an arthroplasty surgery. The burden of the infection is far-reaching, not only to the patient, family and society, but also to the operating surgeon. In most instances, especially for late-presentation or chronic infection, a 2-stage surgery is favoured incorporating surgical debridement with implant removal, followed by antibiotic therapy before a revision arthroplasty surgery is done. However, we here will describe a rare case of a systemic lupus erythematosus (SLE) patient who presented with right knee swelling 4 months after right total knee arthroplasty, which eventually turned out to be Mycobacterium tuberculosis infection. She was successfully treated with debridement and retention of implant, together with commencement of anti-tuberculosis medications.

\section{Case Report}

A 58 years old lady presented to us with painful right knee swelling 4 months after undergoing a total knee replacement in a private hospital. She was known to have systemic lupus erythematosus (SLE) since 1989 and was on low-dose prednisolone and methotrexate. There was however no history of previous tuberculosis (TB) infection, contact with a known TB patient, chronic cough or constitutional symptoms suggesting an occult infection. Prior to the total knee arthroplasty, she presented to the private hospital with history of chronic pain of bilateral knees. The pain gradually worsened over time and resulted in limitation of activities of daily living, including her walking distance. A diagnosis of bilateral knee osteoarthritis was made and following consultation, she agreed and proceeded with the total knee arthroplasty. In retrospect, preoperative workups were normal, including a normal level of erythrocyte sedimentation rate (ESR), C reactive protein (CRP) and total white cell count (TWCC)-which suggested that there was no acute flare of SLE and patient was not having any active infection. Urine microscopy and culture were also clear. Preoperative knee radiographs showed degenerative arthritis changes involving all three compartments of her right knee, with presence of peri-articular osteopenia, which is not uncommon in patients with secondary osteoarthritis.

A cemented, semi-constraint, posterior-stabilised right total knee replacement was subsequently done for her. Her post-opera-

. 
tive recovery was uncomplicated, and the patient started ambulating with walking frame and began physiotherapy as early as day 3 after the surgery. At the 6-week follow up, her wound was completely healed, and her right knee range of motion was almost 0 until 90-degree flexion. Upon presentation to us at 4 months after the arthroplasty, she complained of painful right knee swelling for 3 days prior to admission. Examination revealed a diffusely swollen right knee with small outpouching on the medial side. The right knee was generally warm but non-tender on palpation. The range of motion was greatly limited due to the swelling. The X-rays showed no signs of implant loosening (Figure 1). ESR was $23 \mathrm{~mm} / \mathrm{h}$ while both CRP and TWCC were normal $-4 \mathrm{mg} / \mathrm{L}$ and $7.6 \times 10^{9} / \mathrm{ml}$ respectively. She was started on empirical antibiotic of intravenous ceftriaxone for about a week before the blood parameters were repeated. The subsequent reading of ESR was $25 \mathrm{~mm} / \mathrm{h}$, while CRP was $11 \mathrm{mg} / \mathrm{L}$ and TWCC was $9.0 \times 10^{9} / \mathrm{ml}$. The swelling showed no signs of regression; thus, it was decided that she was to undergo arthrotomy washout of right knee with change of polyethylene insert. Intraoperatively, reactive synovitis was evident, with presence of synovial hypertrophy (Figure 2). There were also pockets of haematoma containing about $300 \mathrm{mls}$ of blood clots. There was no slough or pus present, and neither of the total knee replacement components was found to be loose.
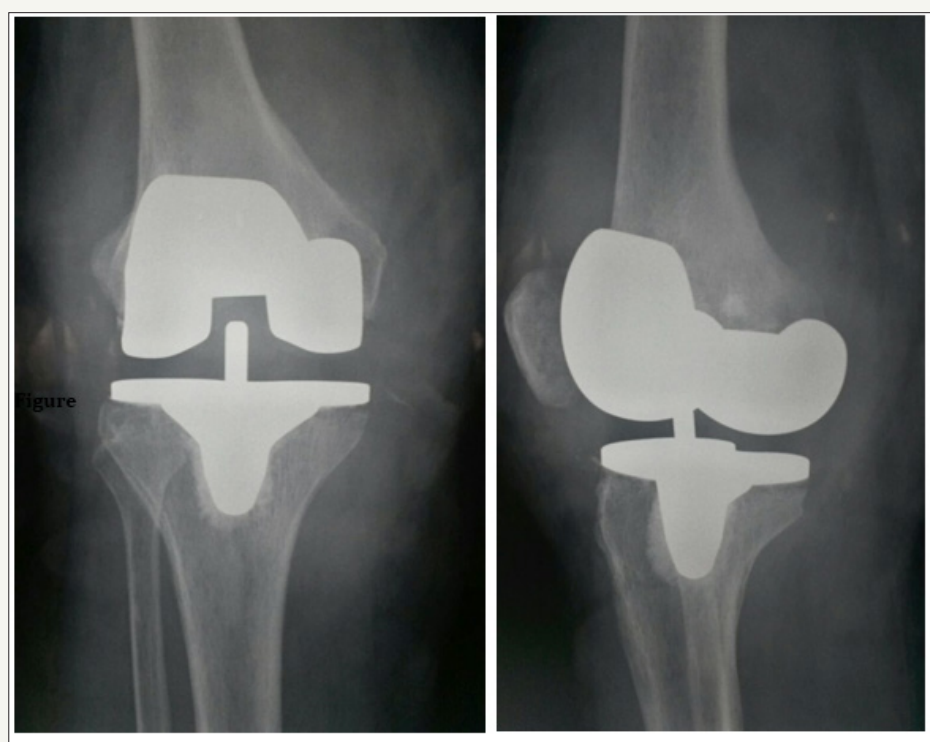

Figure 1: X-ray of the right knee (ap and lateral view) taken on the day of patient's presentation. There is no loosening of the components although the soft tissue shadows were suggestive of soft tissue reaction, correlating with the presence infection. There is no periosteal elevation to osteomyelitis changes visible.

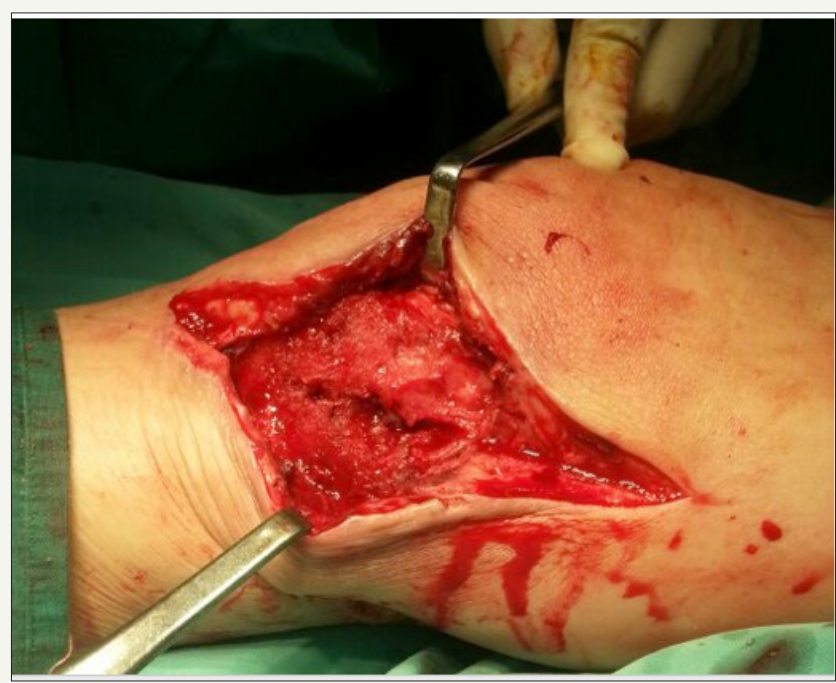

Figure 2: Intraoperative image of the arthrotomy washout and change of insert. There was excessive synovium (synovial hypertrophy) with inflammation suggestive of reactive synovitis.

The synovial tissue and intraarticular fluid and swab sent for culture and sensitivity yielded no growth of organism. Intravenous ceftriaxone was continued for another 2 weeks after the surgery while waiting for the result of the histopathological examination
(HPE) of the synovial tissue. However, during this period, her knee became swollen again with re-formation of the abscess at the medial joint line which subsequently forming a draining sinus (Figure 3). She underwent another debridement with intraoperative findings 
almost like the previous surgery. This time, a specific culture for Mycobacterium tuberculosis was sent and requested. Microscopic examination later revealed presence of acid-fast bacilli with granulomatous reaction (Figure 4). The synovial tissue culture isolated Mycobacterium tuberculosis after 4 weeks. The patient was referred to the Infectious Disease Team and was started on antituberculosis therapy. The medical therapy consisted of Akurit-4 (rifampicin, pyrazinamide, ethambutol and isoniazid, which was commenced for 8 weeks. The subsequent regime consisted of Akurit (rifampicin and isoniazid) was planned for at least another 9 months. Subsequent decision on the anti-tuberculosis therapy will be made based on patient's clinical response. Her condition rapidly improved after the commencement of anti-tuberculosis therapy.
The infectious symptoms of her right knee progressively achieved resolution within 6-8 weeks. Her wound dries off and healed within 8 weeks (Figure 5). She was able to start her physiotherapy session again after 4 weeks. Subsequently at 10 weeks after the commencement of anti-tuberculosis therapy, she was able to ambulate without support or assistance. She was able to achieve right knee flexion up until $90^{\circ}$. Blood parameters consisted of ESR and CRP were found to be normalise and stabilise started at the $10^{\text {th }}$ week. The latest radiograph repeated on her latest follow up at 7 months of after commencement of anti-tuberculosis therapy showed that there was no loosening of the implant, nor any evidence of osteolysis. She is currently still under regular annual follow up.

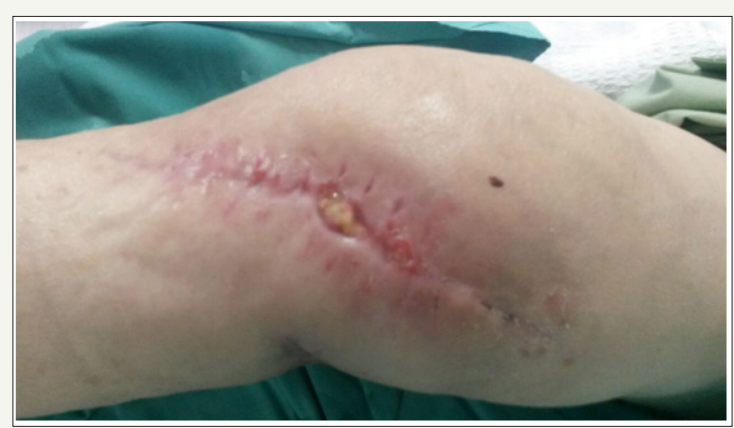

Figure 3: Right knee at 2 weeks after the arthrotomy washout and change of insert. The patient developed a pus-draining sinus on the wound at medial aspect of the knee joint. The area was fluctuant, and the surrounding skin was inflamed.
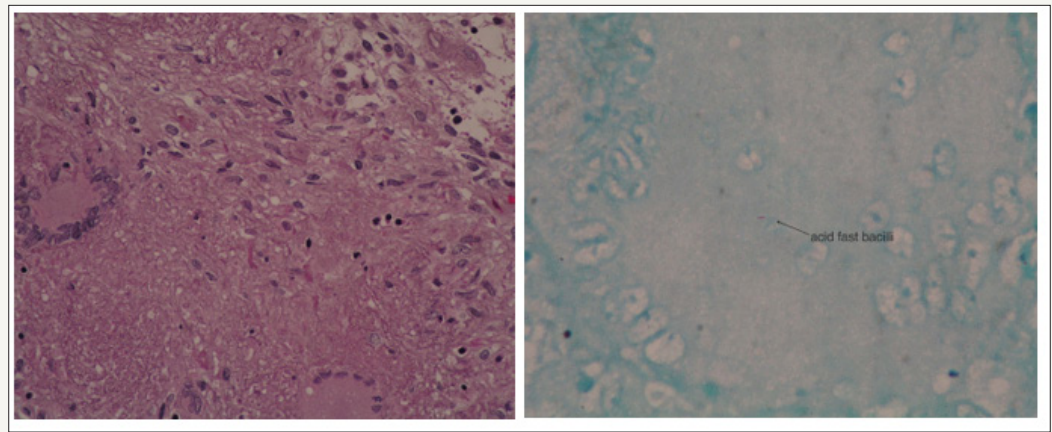

Figure 4: Histopathological examination of the synovial tissue obtained from the debridement (a)Showing dense lymphocytic infiltration with presence of granuloma, together with polymorphs and epitheloid cells while (b)Ziehl-Nielsen stain, an acid-fast bacilli was isolated and identified. This is at paramount importance in diagnosing TB infection in this patient.

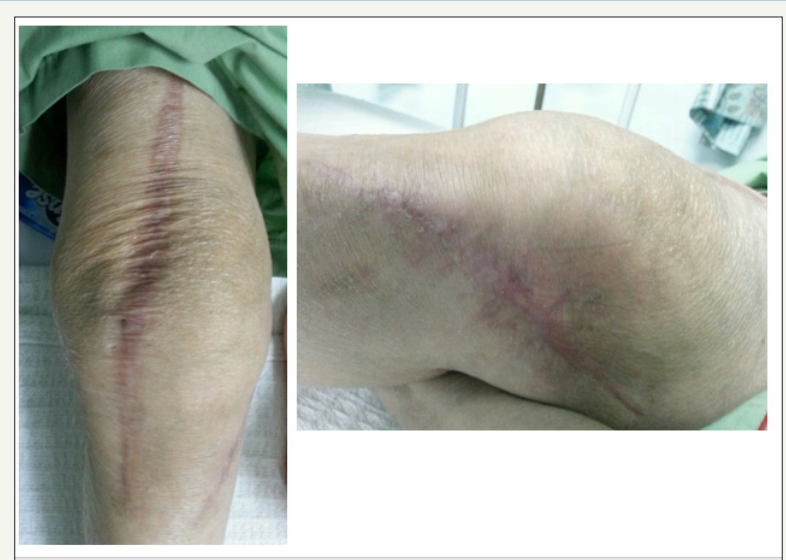

Figure 5: Right knee at 8 weeks after commencement of anti-tuberculosis therapy. There was marked improvement of the soft tissue and skin condition after the commencement of anti-tuberculosis therapy. The wound was well-healed, and the range of motion has improved. 


\section{Discussion}

Despite of the resurgence of tuberculosis infection in this country and this region, periprosthetic joint infection with Mycobacterium tuberculosis is still rare [1,2]. This case highlights a spectrum of possibility and the challenges on managing them accordingly. Apart from post-operative TB infection following a total knee replacement, the association of TB infection in SLE patients and the possibility of reactivation of pre-existing osteoarticular TB could not be discounted. TB is known as "the great mimicker", and its unpredictability might lead to calamitous effects on the patients affected [2]. Apart from the sporadic cases reported throughout the years, one retrospective review by Berbari et al. [1] involving 2116 cases of prosthetic joint infection within a 22-years period found only 7 cases attributed to Mycobacterium tuberculosis infection. Therefore, a post-operative infection of tuberculosis is considered a rarity. However, in this patient who received antimetabolite (methotrexate) and steroid (prednisolone) for SLE, the risk of obtaining prosthetic joint infection was significantly high [3]. Studies have shown a convincing association between SLE and TB infection. Feng et al. [4] found that in a cohort of 311 SLE patients, $5 \%$ or 16 patients was diagnosed with TB. A review by Hodkison et al. [5] showed that TB was diagnosed in 97 out of 568 SLE patients, with incidence of osteoarticular TB was found to be statistically higher than the control group. This raises question on whether this patient might have co-morbid TB infection, given a strong association of SLE and TB. As ESR is usually increased in either case of SLE and TB, it could not be a reliable indicator to separate between these two.

The possibility of pre-existing or reactivation of dormant osteoarticular TB in this patient should also be given a thought. The presence of peri-articular osteopenia on the preoperative X-rays and synovial hypertrophy during the surgery would be supporting this. In retrospect, it would be helpful if synovial tissue was sent for HPE during the primary arthroplasty surgery. TB is still one of the leading underdiagnosed diseases - therefore possibility of pre-existing TB should not be discounted. The diagnosis of TB infection, especially osteoarticular TB or periprosthetic TB, in majority of cases is also often delayed. This is mainly contributed by 3 major factors: the insidious character of the disease itself, the need for culture or biopsy to confirm the diagnosis, and the absence of 'typical' symptoms and causative exposure [2,6]. As seen in this case, there was no strong suggestive hint that could lead to an earlier diagnosis of TB. Therefore, a high index of suspicion is invaluable. It needs to be emphasised that Mycobacterium tuberculosis is isolated in about $60-89 \%$ of the cases, whereas only $0-5 \%$ of joint fluid examined will stain acid fast bacilli $[7,8]$.
This patient is one of the rare examples when both investigations yield positive results. The rapid improvement seen in this patient after commencement of anti-tuberculosis therapy showed that medical therapy remains as the mainstay of treatment. In cases of chronic prosthetic joint infection that occur more than 4 weeks after the primary arthroplasty, a two-stage revision arthroplasty remains as gold standard [9]. This patient was however subjected to an exchange of polyethylene insert with components retention, followed by intravenous antibiotics prior to obtaining the culture and HPE result. As the clinical parameters improved after the start of anti-tuberculosis therapy, the long-term justification of such decision remains to be judged and may only be concluded at the ending of the anti-tuberculosis therapy.

\section{Conclusion}

In conclusion, although prosthetic joint infection by Mycobacterium tuberculosis is rare, the possibility of its postoperative infection should not be excluded especially in immunocompromised patients. A higher index of suspicion coupled with assistance from the appropriate investigations and meticulous observation of patient's condition might be paramount to patient's outcome. Surgical debridement together with the anti-tuberculosis therapy should be initiated as early as possible to ensure a better outcome for the patient.

\section{References}

1. Berbari EF, Hanssen AD, Duffy MC, Steckelberg JM, Osmon DR (1998) Prosthetic joint infection due to mycobacterium tuberculosis: A case series and review of the literature. Am J Orthop 27(3): 219-227.

2. Watts HG, Lifeso RM (1996) Tuberculosis of bones and joints. J Bone Joint Surg Am 78(2): 288-298.

3. Pulido L, Ghanem E, Joshi A, Purtill JJ, Parvizi J (2008) Periprosthetic joint infection: The incidence, timing, and predisposing factors. Clin Orthop Relat Res 466(7): 1710-1715.

4. Feng PH, Tan TH (1982) Tuberculosis in patients with systemic lupus erythematosus. Annals of the Rheumatic Diseases 41(1): 11-14.

5. Hodkison B, Musenge E, Tikly M (2009) Osteoarticular tuberculosis in patients with systemic lupus erythematosus. Q J Med 102(5): 321-328.

6. Nathanson E, Nunn P, Uplekar M, Floyd K, Jaramillo E, et al. (2010) MDR tuberculosis-critical steps for prevention and control. N Engl J Med 363(11): 1050-1058.

7. Hopewell PC (2005) Overview of clinical tuberculosis. In: Bloom BR (Ed.), Tuberculosis. American Society for Microbiology, Washington, USA, pp. 25-46.

8. Teo SH, Teh KK, Azura L, Ng Y (2011) The great mimic again? a case of knee tuberculosis. Malaysian Orthopaedic Journal 5(3): 32-34.

9. Tsukayama DT, Goldberg VM, Kyle R (2003) Diagnosis and management of infection after total knee arthroplasty. J Bone Joint Surg Am 85(1): 75-80. 
Creative Commons Attribution 4.0 International License

For possible submissions Click Here

Submit Article
OPROJ

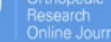

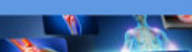

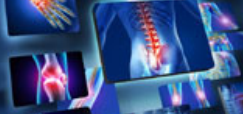

की
Orthopedic Research Online Journal

\section{Benefits of Publishing with us}

- High-level peer review and editorial services

- Freely accessible online immediately upon publication

- Authors retain the copyright to their work

- Licensing it under a Creative Commons license

- Visibility through different online platforms 